\title{
Advances in Laser Driven Accelerator R\&D
}

\author{
Wim Leemans \\ Lawrence Berkeley National Laboratory, 1 Cyclotron Road, MS 71-259, Berkeley, CA 94720, \\ wpleemans@lbl.gov
}

\begin{abstract}
Current activities (last few years) at different laboratories, towards the development of a laser wakefield accelerator (LWFA) are reviewed, followed by a more in depth discussion of results obtained at the L'OASIS laboratory of LBNL. Recent results on laser guiding of relativistically intense beams in preformed plasma channels are discussed. The observation of mono-energetic beams in the $100 \mathrm{MeV}$ energy range, produced by a channel guided LWFA at LBNL, is described and compared to results obtained in the unguided case at LOA, RAL and LBNL. Analysis, aided by particle-in-cell simulations, as well as experiments with various plasma lengths and densities, indicate that tailoring the length of the accelerator has a very beneficial impact on the electron energy distribution. Progress on laser triggered injection is reviewed. Results are presented on measurements of bunch duration and emittance of the accelerated electron beams, that indicate the possibility of generating femtosecond duration electron bunches. Future challenges and plans towards the development of a $1 \mathrm{GeV}$ LWFA module are discussed.
\end{abstract}

\section{INTRODUCTION}

As reported at the Advanced Accelerator Concepts (AAC) 2004 workshop, since the 2002 Workshop, tremendous progress has been made in the development of laser driven wakefield accelerators. For nearly a decade, experiments have been carried out by many groups around the world, in which intense laser pulses are focused onto a neutral gas. Under the "right" conditions, relativistic electron beams were produced which, just like the first reported results on laser wakefield accelerated bunches [1, 2], had 100\% energy spread. Over the years, the quality of these bunches improved, and beams were produced with "smaller" lasers, capable of operating at higher repetition rate. Laser pulse shape effects were studied [3,4], and applications were explored such as radio-isotope production [5, 6, 7] and $\mathrm{THz}$ radiation generation [8, 9]. At AAC2002, experiments were reported, in which beams containing electrons with energy up to $200 \mathrm{MeV}$ had been produced, using a $30 \mathrm{TW}, 30$ fs laser [10]. At the CLEO2004 meeting, production of electrons with energy as high as $350 \mathrm{MeV}$ with the petawatt VULCAN laser at Rutherford Appleton Laboratory (RAL) (delivering $160 \mathrm{~J}$ in $650 \mathrm{fs}$ ) was reported [11]. Whereas the end point energy of the distribution had increased to several 100's MeV, all experiments reported electron distributions that had $100 \%$ energy spread, with a small amount of charge at these high energies $(<<1 \%)$.

At the most recent AAC 2004 workshop however, three different groups (L'OASIS at LBNL, LOA-France and RAL-UK) presented results on the observation of narrow energy spread beams, with $100 \mathrm{MeV}$-level mean energies, containing large amounts of 
charge (order $10^{9}$ electrons/bunch). ${ }^{1}$ All three experiments utilized a short pulse laser system, and extended the distance over which the laser beam remains at high intensity. The LOA and RAL groups utilized a large laser spot (18 and $25 \mu \mathrm{m}$, respectively), i.e., long Rayleigh length of the laser to match the length of the gas jet. The LBNL group utilized plasma channeling technology, which permits the propagation of tightly focused laser beams (e.g. $6 \mu \mathrm{m}$ ) over more than 10 Rayleigh lengths [12]. These results will be discussed (and interpreted) below. Details of the different experiments from the various groups can also be found in other papers in the AAC2004 proceedings.

The importance of the accelerator length has been discussed theoretically for many years. As an example, during the 1995 Kardamyli Workshop on second generation plasma based accelerators, design studies were carried out in the laser guiding and acceleration working group, for a $1 \mathrm{GeV}$ laser wakefield accelerator [13]. Scaling laws were derived for the energy gain of an accelerated electron for different acceleration distances. Motivated by the fact that most experiments are to a significant degree dictated by the availability of the particular laser system, expressions were derived in terms of laser and plasma parameter choices, i.e., from the standpoint of the laser builder and experimentalist. The main conclusion of the study was that extending the propagation of a laser pulse beyond its natural diffraction distance or Rayleigh length, $Z_{R}$, up to the dephasing distance [14], was an essential element of a future LWFA. Much higher net energy gains can be realized for the same amount of input laser power, by extending the acceleration distance beyond $Z_{R}$. The key design equations will be reviewed in Sec. 2 .

Guiding concepts relying on the use of preformed channels are being studied by several groups around the world, including LBNL [15, 12], University of Maryland [16], University of Texas at Austin [17], UCLA [18], Naval Research Laboratory [19], Oxford University-UK [20], Japan [21], IST Lisbon-Portugal [22], Taiwan [23] and France [24]. At LBNL, using the ignitor-heater concept [15], we have recently produced plasma channels that have guided, to our knowledge, the highest peak power in a preformed channel [12]. This result demonstrates the ability of plasma channels to guide laser beams, at intensities relevant for particle acceleration, over many $Z_{R}$. After a brief introduction, experimental results from the L'OASIS group on laser guiding will be summarized in Sec. 3. They are described in more detail by Geddes et al. in these proceedings [25].

In addition to guiding at high intensity, the production of high quality electron beams from these plasma channels was observed [12]. As will be discussed, these results are consistent with extending the acceleration distance up to the dephasing distance, which is the distance it takes a trapped electron to outrun a plasma wave that propagates at a subluminous phase velocity. Matching the accelerator length to the dephasing length can alternately be met, though less efficiently, without a channel. A short gas jet can be used at the cost of reduced final energy. Self guiding can be used in longer jets, but this is much less stable than a channel. A large laser spot can also be used to obtain a long $Z_{R}$, at the cost of efficiency (higher laser power needed to obtain high energy

\footnotetext{
${ }^{1}$ The narrow energy spread observations at LBNL and RAL were also presented at CLEO 2004.
} 
electron). The impact of dephasing on the accelerator has been studied experimentally at the L'OASIS facility, by using gas jets of variable length and density, without channeling [26]. Similar results on the importance of the dephasing distance have been reported at the AAC2004 Workshop by the RAL group. Experimental results from the LBNL group will be discussed, and compared with the French and British results in Sec. 4. The LNBL results are also discussed in detail in these proceedings by Geddes et al. [25].

Whereas mono-energetic electron bunches containing hundreds of picoCoulomb of charge have now been observed, the shot-to-shot stability is insufficient for most applications. The uncontrolled nature of the trapping process is believed to contribute to the fluctuations. Experiments are underway to control this trapping and acceleration process at the Naval Research Laboratory using the LIPA method (Laser Ionization Ponderomotive Acceleration) [27], at the University of Michigan using the LILAC method (Laser Injection Laser Acceleration) [28], and at LBNL using the CPI method ( Colliding Pulse Injection) [29, 30, 31]. Progress on the CPI method is summarized below in Sec. 5, and presented in more detail in these proceedings by Nakamura et al. (these proceedings) $[32]$.

Diagnostics for studying the wakefield excitation, laser propagation in the plasma, and electron bunch properties are being developed. Following the successful demonstration of Fourier domain interferometry techniques for measuring laser wakefields in uniform plasmas [33, 34], the University of Texas Austin group is now developing techniques for studying laser propagation in channels, using femtosecond pump-probe techniques [17]. This permits detection of subtle changes in laser mode distortion, as well as measurement of residual un-ionized plasma. The presence of incompletely ionized gas may perturb the channel's guiding properties.

Methods for measuring the bunch duration of laser accelerated electron beams have also recently been studied. One of these relies on a cross-correlation between the accelerated electron beam and a second laser. Through its ponderomotive pressure, the laser deflects the electron bunches when they exit the plasma. This method has been under development at the University of Michigan [35]. A method that relies on measuring the coherently radiated $\mathrm{THz}$ spectrum of the electron bunch has been studied at LBNL, and will be discussed in more detail in Sec. 6. Details can also be found in these proceedings by van Tilborg et al. [36]. The radiation was emitted when the electron bunch crossed the plasma-vacuum boundary. Multi-shot interferometric measurement of the spectrum, obtained using a Michelson interferometer, indicates bunch durations on the order of 50 fs or shorter [36]. A single shot electro-optic sampling method is being implemented, for measuring the bunch duration on a shot-to-shot basis.

Emittance measurements on broad energy spread beams were recently made using a pepper pot technique [37]. The emittance (normalized) was found to be energy dependent, and on the order of $3 \pi \mathrm{mm}$-mrad for a $55 \mathrm{MeV}$ slice of the broad energy distribution, but higher than $35 \pi \mathrm{mm}$-mrad for electrons less than $20 \mathrm{MeV}$. In general, for dense, high charge beams with large energy spread, space charge effects and energy spread can considerably affect the emittance and bunch duration, during propagation in vacuum $[38,39]$. Care must be exercised when interpreting measurements of these quantities, as they can be position dependent and complex to interpret for the low energy electrons. 
This is much less of an issue for the higher energy (100 MeV-level), percent level energy spread beams, such as produced in the channel guided LWFA, or with the larger spot size method. However, detailed phase space measurement techniques, for example, the tomographic methods used at BNL [40] need to be developed with femtosecond and micron resolution to enable careful study of the properties of laser accelerated bunches.

The progress on the development of laser driven accelerator has been the result of progress in, and availability of, high peak power short pulse laser systems, that rely on chirped pulse amplification, and that are able to provide multiple laser beams with controlled parameters. An example of such a laser system, the multi-terawatt multibeam L'OASIS laser at LBNL, is discussed in the AAC2004 proceedings by Toth et al. [41]. The L'OASIS laser system is currently capable of delivering up to five different synchronized laser beams onto the targets, with one of the beams having peak power as high as 10-15 TW, at repetition rates up to $10 \mathrm{~Hz}$. An additional laser amplifier arm is under commissioning, and is expected to deliver 3-4 $\mathrm{J}$ in a 30-50 fs duration laser pulses (i.e., 50-100 TW) at $10 \mathrm{~Hz}$. The laser beams are all delivered to a radiation shielded experimental area, and experiments are conducted from a remote control room. Data from all diagnostics are acquired on a central control system, and many of the experimental system components can be remotely computer controlled. The setup for the various LBNL experiments that will be reviewed, is shown in Fig. 1, and used laser beams produced by the L'OASIS laser system.

The last Section is dedicated to a short summary of the most important recent results, and a look at the challenges ahead for the development of stable, GeV-class laser driven accelerators.

\section{BASIC EQUATIONS}

During the 1995 Kardamyli design study [13], the choice was made to operate the accelerator in the standard laser wakefield regime, where the laser pulse duration $\tau_{L}$ is on the order of the plasma wavelength $\lambda_{p}$. In addition, the laser power was assumed to be less than the critical power for self-focusing in a plasma. The motivation for these assumptions was that a laser driven accelerator should be controllable, i.e., not operate in a regime where instabilities can severely affect the laser propagation and particle acceleration. The maximum electron energy gain can then be calculated by integrating the longitudinal electric field strength over the distance over which the particle will experience the accelerating phase of the field. The basic expressions used in this paper for the plasma wave amplitude, and hence longitudinal field, can be found in [14, 13]. Two different cases can now be considered. In the first case, one assumes that the laser is unguided and that the acceleration distance is given by the Rayleigh length $Z_{R}=\pi w_{0}^{2} / \lambda$, where $w_{0}$ is the laser spot size and $\lambda$ is the laser wavelength. The maximum electron energy gain is then given by

$$
\Delta E_{\text {diff }}[M e V] \simeq 580\left(\lambda / \lambda_{p}\right)\left(1+a_{0}^{2} / 2\right)^{-1 / 2} P[T W]
$$




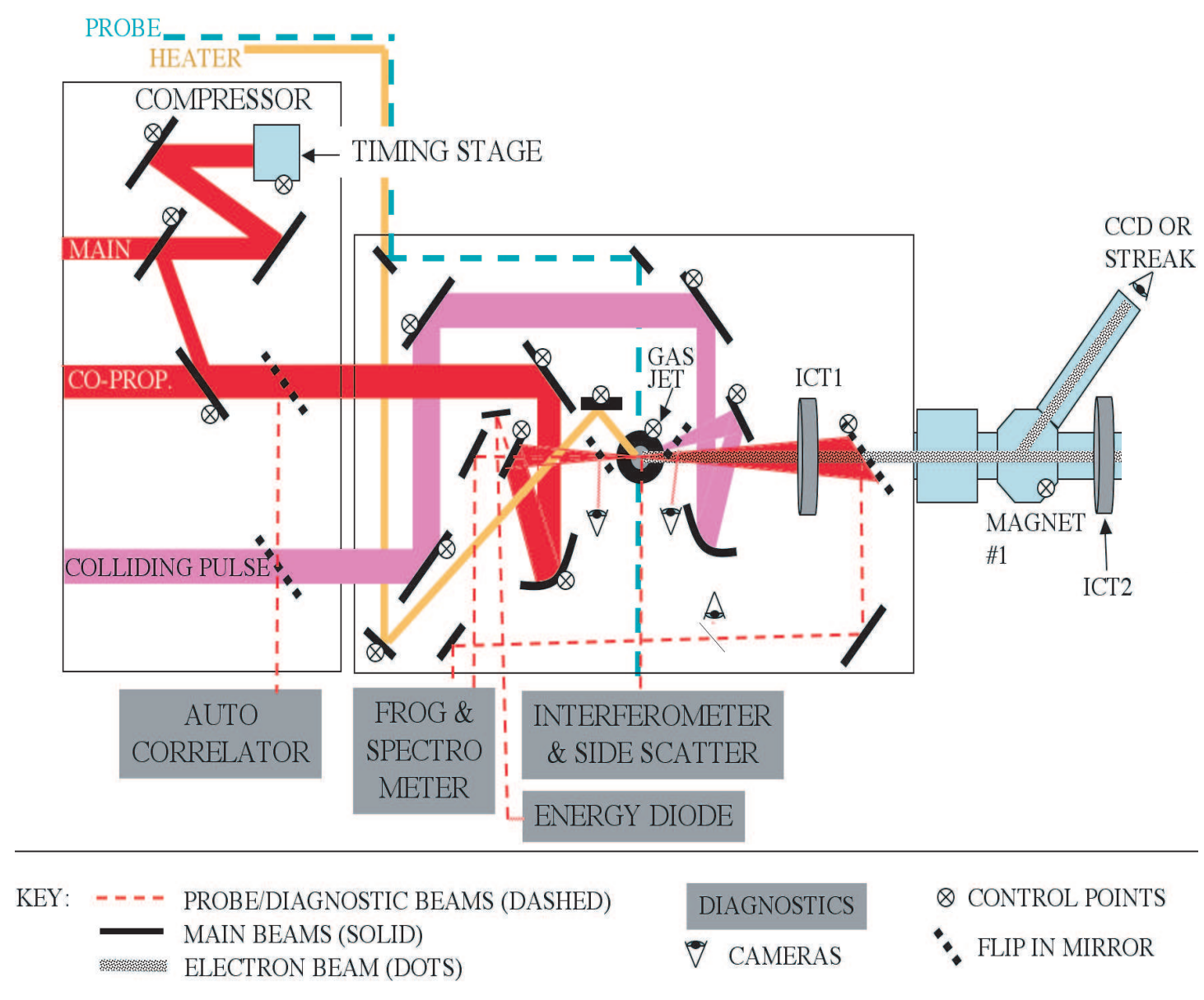

FIGURE 1. Schematic of the experimental setup used for the different experiments described in this paper.The laser beams are produced by the L'OASIS system. Plasma density profiles are measured using a frequency doubled low power probe beam. For single beam experiments only the "main"or drive beam is used, that can reach up to $12 \mathrm{TW}$ peak power. Guiding experiments utilize an additional two beams: the ignitor beam $(50 \mathrm{fs}, 15 \mathrm{~mJ})$ that co-propagates with the main drive beam and the heater beam $(200 \mathrm{ps}, 150$ $\mathrm{mJ}$ ) which is focused onto the plasma using a cylindrical mirror. Two beam colliding pulse experiments utilize one additional pulse $(0.1-0.3 \mathrm{~J}, 50 \mathrm{fs})$ that intersects the drive pulse at a $150^{\circ}$ angle. The high peak power laser beams are focused, using off-axis parabolic mirrors, onto a high pressure pulsed gas jet, operating with about 70 bar helium backing pressure. All beam lines have adjustable delay lines for synchronization, and have their own compressor, except for the heater beam which is used uncompressed. Pulse durations and shapes are measured with a second order autocorrelator, and a polarization based Frequency Resolved Optical Gating system, respectively. The drive laser's energy on target is monitored using a diode, and forward and backward scattered radiation is measured with an optical spectrometer. An integrating current transformer (ICT) is used to measure the charge per bunch of the electron beam. A $55^{\circ}$ dipole magnet permits energy distribution measurements up to $92 \mathrm{MeV}$ with about $1 \%$ resolution and a range of $25 \%$ around a chosen central momentum. The magnet current is adjusted to choose the central momentum. Higher energies (up to $150 \mathrm{MeV}$ ) are measured by operating the spectrometer at a $5^{\circ}$ angle, at the cost of resolution (10\% level). Several $\gamma$-ray and neutron detectors are positioned around the experiment to monitor ionizing radiation. Terahertz radiation is collected with an off-axis parabola (not shown) and transported to a liquid helium cooled bolometer (energy measurement) and Michelson interferometer (spectral measurement). 
where $a$ is the normalized vector potential given by $[14] a \simeq 8.6 \times 10^{-10} \lambda[\mu \mathrm{m}] \mathrm{I}^{1 / 2}\left[\mathrm{~W} / \mathrm{cm}^{2}\right]$, with $I$ the laser intensity.

In the second case, one assumes that the laser is guided over a distance equal to the dephasing distance $L_{d e p h}$, which is assumed to be long compared to $Z_{R}$. The dephasing distance (to slip a non-linear plasma length $\lambda_{N p} / 2$ ) is given by [14]

$$
L_{d e p h}=(1 / 2)\left(\lambda_{p}^{3} / \lambda^{2}\right) \times \begin{cases}1, & a_{0}^{2} \ll 1 \\ (\sqrt{2} / \pi) a_{0} / N, & a_{0}^{2} \gg 1\end{cases}
$$

where $\mathrm{N}$ is the number of plasma periods. Three dimensional effects can decrease this distance by a factor of 2 and, to be in the 1-D limit, it is assumed that $\pi w_{0} / \lambda_{p} \approx 1$. Extending the propagation distance of a focused laser beam can be done by using a preformed plasma, with a radial density profile suitable for guiding, i.e. minimum on axis $[42,43]$. The maximum electron energy is then given by

$$
\Delta E_{d e p h} \simeq 60\left(\lambda_{p} / w_{0}\right)^{2} P[T W] \times\left\{\begin{array}{ll}
1, & a_{0}^{2} \ll 1 \\
(2 / \pi), & a_{0}^{2} \gg 1
\end{array},\right.
$$

neglecting pump depletion effects [44].

From these scaling laws it becomes apparent that if $L_{d e p h}>\pi Z_{R}$, which implies $\lambda_{p}>\lambda$ (or $n<n_{c}$ where $n_{c}$ is the critical density which is a necessary condition for propagation of the laser), extending the acceleration distance beyond $Z_{R}$ can result in $\mathrm{GeV}$ electron beams, using laser systems that produce peak powers on the order of a few tens of TW. As an example, consider the case of a $100 \mathrm{TW}$ laser beam $(5.5 \mathrm{~J}$ in $55 \mathrm{fs}$ ) operating at $\lambda=800 \mathrm{~nm}$ and focused to a spot size of $18 \mu \mathrm{m}$. The normalized vector potential is $a=3$ and the intensity is $I_{L}=1.9 \times 10^{19} \mathrm{~W} / \mathrm{cm}^{2}$. Matching the pulse duration to the plasma wavelength $\left(L_{L}=\lambda_{p} / 2\right)$ then requires a plasma density $n_{0}=$ $10^{18} \mathrm{~cm}^{-3}$. In the unchanneled case, the maximum energy gain is $\Delta E_{\text {diff }}=600 \mathrm{MeV}$ in $L_{a c c}=\pi Z_{R}=4 \mathrm{~mm}$ versus $\Delta E_{d e p h}=12.8 \mathrm{GeV}$ in $L_{d e p h}=3.8 \mathrm{~cm}$. This simple description does not include pump depletion of the laser beam, due to energy transfer into the plasma and particle beam, and is therefore an overestimate. Pump depletion is discussed in these proceedings by Esarey et al. [44]. The key challenge then is to develop plasma channels that can guide relativistically intense laser beams $(a>>1)$. In the next Section, recent results will be discussed, that demonstrate that radially shaped plasmas can indeed guide intensities relevant to large amplitude wake excitation and particle acceleration.

\section{GUIDING RELATIVISTIC INTENSITIES IN PREFORMED PLASMA CHANNELS}

As discussed in the previous section, the ability to guide intense laser pulses over many $Z_{R}$, is an essential element of a high energy LWFA. Guiding of short laser pulses in plasma channels was first demonstrated in hydrodynamically formed plasma channels 
$[42,43]$, produced by focusing a relatively intense beam with an axicon lens. In these pioneering experiments, high Z-gases were used to facilitate the ionization process. Heating of the ionized plasma through inverse Bremsstrahlung led to a high temperature plasma filament. Through hydrodynamic expansion, plasma density profiles were produced, that had a minimum on axis, and were suitable for guiding laser pulses. High Z-gases are however susceptible to further ionization when used with ultra-high intensity lasers, and therefore, a method was needed to allow the use of low Z-gases. By separating out the ionization and heating phase of the channel formation, channels were produced in hydrogen gas with the ignitor-heater method [15]. The advantage of operating with hydrogen is that it can be fully ionized at intensities on the order of $10^{14} \mathrm{~W} / \mathrm{cm}^{2}$. Laser pulses were guided in these channels at intensities on the order of $10^{17} \mathrm{~W} / \mathrm{cm}^{2}$ (maximum intensity in 1998 of the L'OASIS laser), too low for exciting large amplitude plasma waves.

Most recently [12], channeling at relativistic intensities was realized with the 10 TW L'OASIS laser. Preformed guiding channels were created using a variation of the ignitorheater method [15] (see Fig. 1). A plasma was formed in a $2.5 \mathrm{~mm}$ long supersonic $\mathrm{H}_{2}$ gas jet with an atomic density of $3-4 \times 10^{19} \mathrm{~cm}^{-3}$, by an ignitor pulse $(15 \mathrm{~mJ}, 60 \mathrm{fs})$ that is co-axial with the drive pulse (instead of orthogonal to as in the original implementation), then heated by a heater pulse (150mJ, 250ps). Hydrodynamic expansion of the plasma formed a channel $[42,43]$, that guided a relativistically intense drive pulse that was focused at the entrance to the channel. The drive pulse $(500 \mathrm{~mJ}, 55 \mathrm{fs})$ was focused to a spot of 7-8.5 $\mu \mathrm{m}$ FWHM resulting in a laser intensity of $1.1 \times 10^{19} \mathrm{~W} / \mathrm{cm}^{2}$ ). Propagation of the laser was monitored with a side interferometer, mode imager CCD, and transmitted light spectrometer. Electrons accelerated by the plasma wake of the drive beam were analyzed using an integrating current transformer (ICT), a phosphor screen, and a magnetic spectrometer.

The ignitor-heater method provided several tuning knobs to tailor the channel properties. Varying the time delay between the heater and drive pulses, energy of the heater pulse and spatial overlap, channels can be created with different radial density profiles. Figure 2 shows an example of mode images of laser spots at $4 \mathrm{TW}(7 \mu \mathrm{m}$ input spot, $7 \times 10^{18} \mathrm{~W} / \mathrm{cm}^{2}$ ). With the channel on, the output spot (b) matches the input (a). The mode imager resolution is restricted by $\mathrm{f} / \#$ constraints in the target chamber, and measures a $12 \mu \mathrm{m}$ FWHM spot size for both input and output. Hence, the guided intensity is between $10^{18}$ and $2.5 \times 10^{18} \mathrm{~W} / \mathrm{cm}^{2}$ with the lower limit set by the $12 \mu \mathrm{m}$ mode imager observation and the upper limit set by the input spot size. In the absence of any plasma, a large mode size consistent with vacuum diffraction is observed (c), and with the gas jet on but the channel off (d) diffraction is increased by ionization effects [45, 46], showing that self guiding alone is insufficient to efficiently guide the beam.

Transmission at $4 \mathrm{TW}$ was $35 \%$, a reduction of one third from the low power case, indicating that substantial power was deposited in plasma waves. The depletion observed, is consistent with particle-in-cell simulations, run with the experimental parameters. The simulations also indicate that a plasma wave averaging $200 \mathrm{GV} / \mathrm{m}$ is excited, in the last $0.5 \mathrm{~mm}$ of guide length. No electrons are self trapped at $4 \mathrm{TW}$, making this an attractive structure for controlled injection experiments [29, 31], as will be discussed below. When 


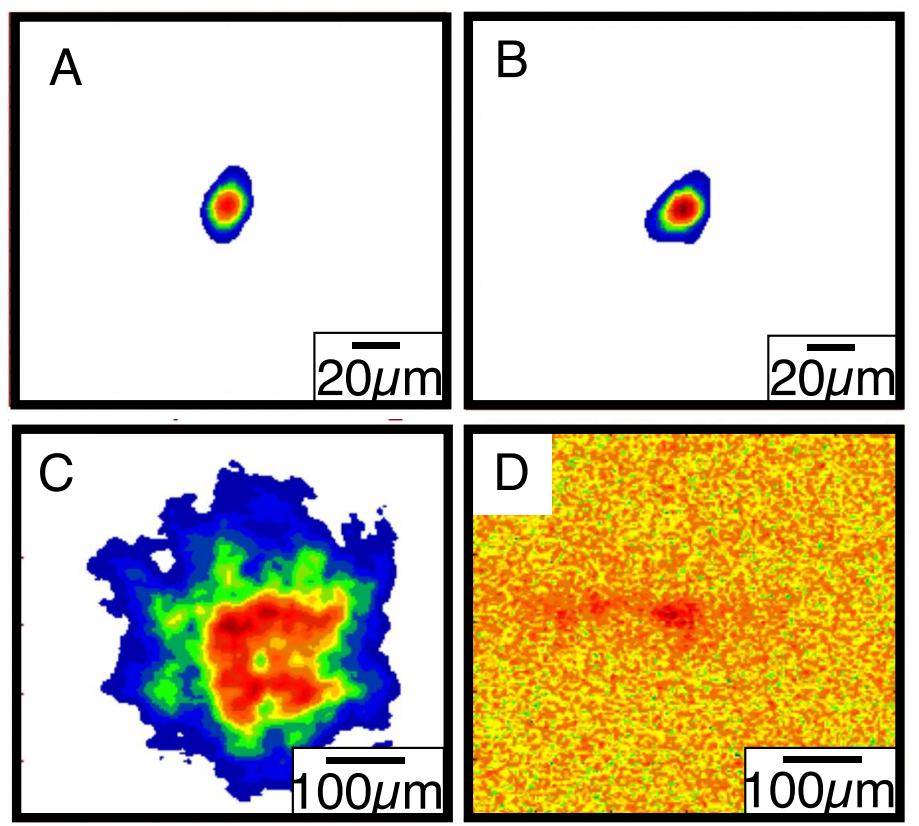

FIGURE 2. Mode images of laser propagation at $4 \mathrm{TW}$, or four times the critical power for self focusing. The guided output mode after $2.5 \mathrm{~mm}$ (10 diffraction ranges) of propagation (B) is indistinguishable from the input mode (A). The effect of the channel can be seen by comparison to vacuum propagation over the same distance where the output mode is severely diffracted (C). Self guiding also does not maintain the spot over this distance due to instability, and the output mode with gas jet on but without the guide is displays enhanced diffraction (D). Note enlarged scale in (C),(D).

increasing the laser power up to the $10 \mathrm{TW}$ level, electron beams were produced with unprecedented properties. This will be discussed next.

\section{PRODUCTION OF LOW ENERGY SPREAD BUNCHES: ACCELERATION TO THE DEPHASING LIMIT}

Using the preformed channels and laser input powers at the 8-10 TW level, electron beams with narrow energy spread were observed [12]. Using the $55^{\circ}$ fine resolution spectrometer, beams containing $2 \times 10^{9}$ electrons with a few percent energy spread around $86 \mathrm{MeV}$ were observed with a divergence on the order of $3 \mathrm{mrad}$. Beams containing $10^{9}$ electrons at energies between $135-150 \mathrm{MeV}$ were observed using the $5^{\circ}$ port of the magnetic spectrometer. An example of a narrow energy spread spectrum is shown in Fig. 3. The normalized geometric emittance, obtained from assuming that the bunch comes from a source approximately the size of the laser spot, is 1-2 $\pi \mathrm{mm}-\mathrm{mrad}$, competitive with state of the art radio-frequency facilities.

To understand the details of the LWFA, particle-in-cell simulations using the code VORPAL [47] were conducted in parameter regimes relevant to the experiments [12]. In these simulations, it is observed that in the first few hundred microns of propaga- 


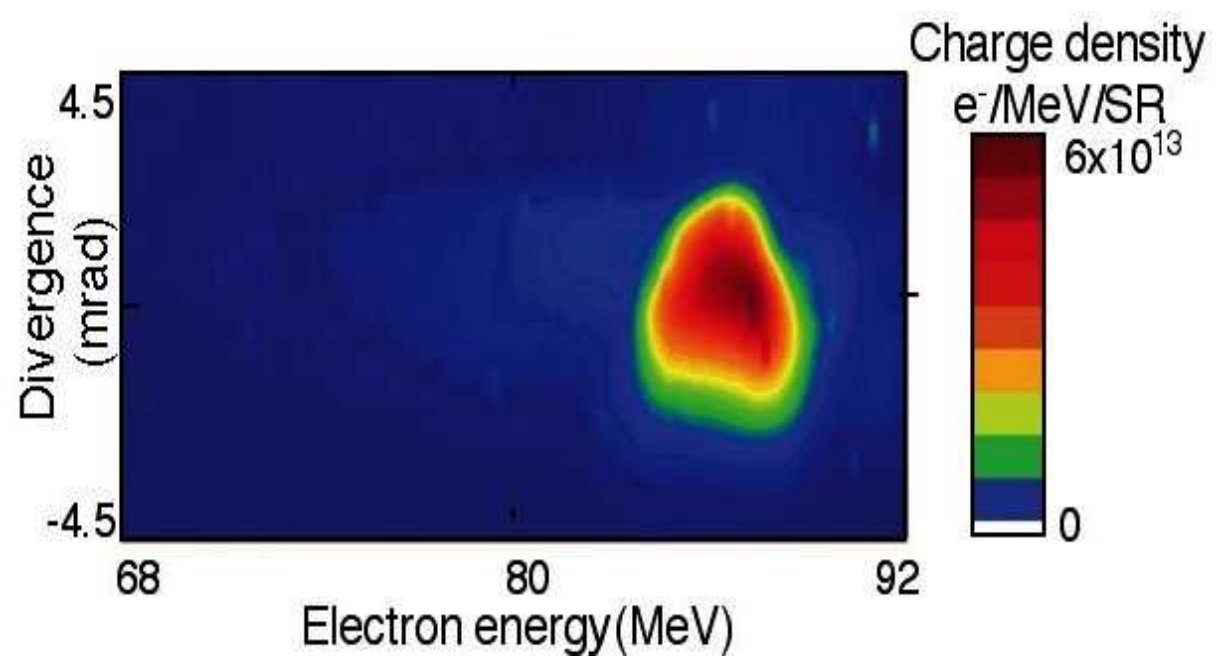

FIGURE 3. Electron energy spectrum of a bunch produced by the channel guided accelerator. The spectrum was obtained by dispersing the electron beam with the $55^{\circ}$ magnetic spectrometer and recording the beam image on a phosphor screen imaged with a high resolution CCD-camera. The energy range covered in this single shot is from $68-92 \mathrm{MeV}$ and shows the appearance of mono-energetic features, here with $3 \times 10^{9}$ electrons in a bunch with energy spread of $4 \% \mathrm{FWHM}$ at $78 \mathrm{MeV}$. In the vertical (non-dispersive) plane, the divergence was near 3 mrad FWHM for this bunch.

tion of the laser pulse in the channel, the wake amplitudes (and hence the amount of trapped particles) are small. As the laser pulse envelope starts distorting through the self-modulation instability, developing features that have rise times on the order of or shorter than the plasma period, a plasma wake is excited, that is large enough to trap and accelerate particles. The first few accelerating buckets can still be relatively low amplitude though. As trapped electrons propagate beyond a dephasing distance, energy is lost and, if the accelerator were terminated here, a broad low energy distribution would be produced. Indeed, the maximum energy gain is dependent on the plasma wave amplitude, and hence, will be lower in the beginning of the channel, where the laser has not become significantly modulated. For longer plasmas however, the self-modulation process can further distort the laser pulse, which then excites a large amplitude wake that can trap large amounts of electrons and accelerate them to high energy. Through an interplay of beam loading by the trapped electrons, and pump depletion, a single accelerating bucket can then be formed. An example of an electron distribution obtained from a PIC code simulation is shown in Fig.4. The acceleration process was terminated when a large amount of electrons reached the dephasing distance. The momentum bunching that occurs at the top of the bucket results in quality electron beams. By carefully controlling the accelerator distance to match the dephasing distance, high energy electron beams with narrow energy spread can hence be obtained.

Reaching the dephasing distance was also put forward by the RAL group as the mechanism behind their observation of narrow energy spread bunches. The LOA group interpreted their result as being consistent with operation in the "bubble" regime [48]. This regime is well known in plasma wakefield accelerators, when using electron beams 


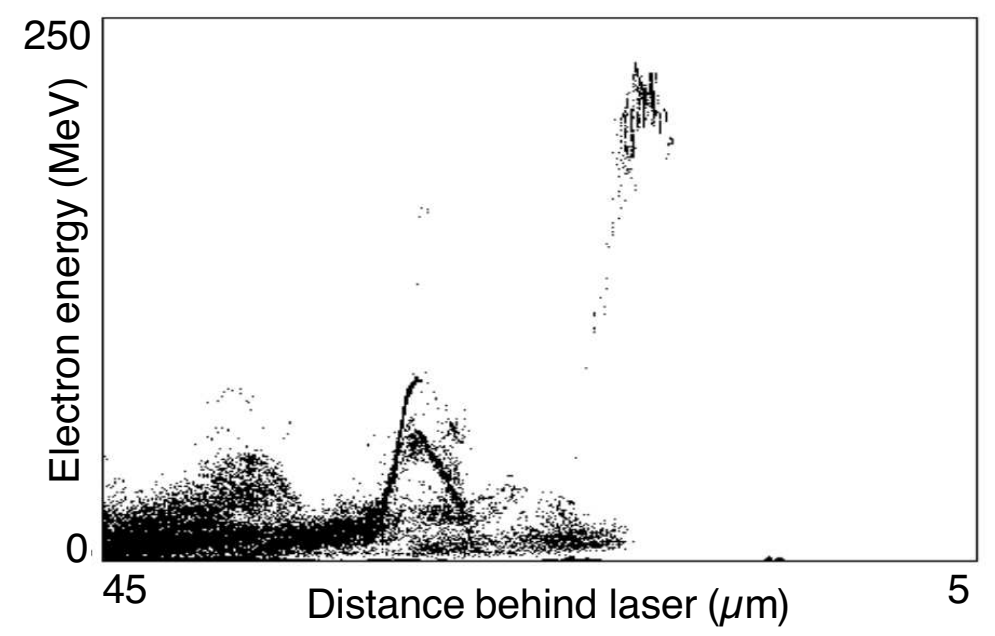

FIGURE 4. Electron energy (MeV) distribution versus longitudinal distance behind the laser pulse $(\mu m)$ obtained from a 2-D particle-in-cell simulation [12] using the code VORPAL [47]. The simulation shows that a high quality bunch can be formed when the acceleration distance is matched to the dephasing length and beam loading by the intense bunches turns off injection. The momentum bunching that occurs when bunches rotate up to the maximum energy level that the accelerating bucket can produce, results in a bunch isolated in phase space. The result is the formation of a low energy spread bunch.

denser than the surrounding plasma. In the case of lasers, it occurs when the ponderomotive potential of the laser is large enough to blow out all plasma electrons, i.e., $a>>1$. Simulations of laser-plasma interaction in this ultra-relativistic regime indicated the generation of quasi-monochromatic bunches [48].

The LOA group suggested, at the AAC2004 Workshop, as evidence for operation in the "bubble" or blow-out regime, the dependence of electron energy spectra on plasma density. Operation at lower density than in their previous experiments gave rise to observation of the structured energy spectra. ${ }^{2}$ Simulation results, reported in Pukhov and Meyer-ter-Vehn (Ref. [48]), used a $30 \mathrm{fs,} 12 \mathrm{~J}$ laser pulse producing $a=10$, in a plasma with density $n_{p}=10^{19} \mathrm{~cm}^{-3}$. This is to be compared to $a \simeq 1.7$ and $n_{p}=6 \times 10^{18} \mathrm{~cm}^{-3}$ as the normalized vector potential and density, respectively, at which narrow energy spread beams were observed in the LOA experiments. Higher density led to more Maxwellian distributions in the experiment. According to the scaling laws presented in Ref. [48], achieving the bubble regime becomes easier, however, at higher density, i.e., requires lower $a$-value. Since the simulation and experimental parameters differ considerably, and the scaling seems to be opposite of what was conjectured in Ref. [48], it is clear that further simulations and experiments are needed to allow closer comparison between them. Studies on propagation of the laser pulses, pump depletion and wake structure are an area of very active research (for example, see Ref. [44]) that could shed light on the detailed physics behind the interplay between wake excitation and laser pulse evolution,

\footnotetext{
${ }^{2}$ It should be noted that, without detectors capable of covering the entire dispersive area of the spectrometer, narrow energy spread features can easily be missed. Such was the case in many of the previous experiments that used diodes for electron detection.
} 
due to self-modulation.

Putting the details of the plasma wake excitation aside, particle dynamics in the accelerating bucket are largely independent of the regime in which the accelerating bucket is created. If the plasma wake has a subluminous phase velocity (due to finite group velocity of the drive laser), such as is generally the case in the absence of density ramps, dephasing between the accelerating particle and plasma wave will occur after some distance. Dephasing (or detuning, to use the language used in Refs. $[48,14]$ ) can then result in momentum bunching, i.e., the production of narrow energy spread bunches.

The next issue for the development of LWFAs that must be addressed, is the stability of the accelerator performance, such as is required in high energy physics and other applications. Shot-to-shot variation in electron energy spectrum causes the amount of charge in a narrow momentum bin to fluctuate. As can be seen from Eqn. 3, electron energy depends on laser intensity, plasma density and plasma length (through the matching with dephasing distance). In regimes were self-modulation of the laser pulse is important, wake amplitudes will also depend on the initial laser pulse shape [3], and hence, pulse shape control is important. However, controlling the laser and plasma initial conditions, and relying on self-trapping, may ultimately still not offer sufficient control over the performance of the accelerator.

\section{COLLIDING PULSE INJECTION}

As was discussed in the previous section, electron beams with narrow energy spread have been produced, for the first time, in a channel guided laser wakefield accelerator. They were also observed in LWFA's where the propagation distance (Rayleigh length or plasma length) was matched with the dephasing distance. However, stability requirements may still require the development of methods for controlled injection.

Several methods have been proposed for triggering injection of electrons in a plasma wake by using one or more additional laser pulses [28, 29, 30, 31]. Here progress will be discussed on implementing a two pulse variation of the colliding pulse optical injection method. As in the original three pulse scheme, this method relies on interfering two laser pulses with each other. A first laser pulse excites a plasma wave in the standard LWFA regime, i.e., sufficiently linear to avoid self-trapped electrons. Time gated, short wavelength ponderomotive buckets, produced when a second laser pulses crosses the first beam, can momentum boost and phase kick electrons that are normally on an untrapped oscillatory orbit, into the accelerating bucket of the plasma wave. The trapped electrons can then be accelerated by the plasma wave of the laser drive pulse. Based on simulations, the two-pulse colliding pulse injection method is expected to produce low emittance ( $1 \pi \mathrm{mm}$-mrad), low energy spread (1\%), $40 \mathrm{MeV}$ femtosecond electron bunches containing $>10^{7}$ electrons per bunch [31].

In the initial experimental configuration of the two pulse CPI method, the beams were crossed at $150^{\circ}$. This avoided having any optics in the path of the electron beam. The L'OASIS laser system at the Lawrence Berkeley National Laboratory [6] produced a drive laser pulse (1 J/pulse) and a colliding pulse $(0.3 \mathrm{~J} /$ pulse) (see Fig. 1). The drive 
and colliding beams were focused onto a $2 \mathrm{~mm}$ long supersonic helium gas jet, backed with up to 70 bar of pressure, to a spot size of $6 \mu \mathrm{m}$ and $12 \mu \mathrm{m}$, respectively. The schematic of the experiment is shown in Fig. 1. The laser beam intensities were $\mathrm{a}_{0}=$ 1.3 and $\mathrm{a}_{1}=0.78$, similar to what was used in the theoretical modeling. The electron beam parameters were measured using an integrating current transformer (ICT) and a magnetic dipole spectrometer. As a rough measure of electron energy, the $\gamma$-ray yield, produced through Bremsstrahlung of the accelerated electrons when stopped in a high $\mathrm{Z}$ material, was also recorded using an ionization detector. The density profile of the plasma was measured using side-on interferometry of the folded-wave type, using a 400 $\mathrm{nm}$ wavelength, $50 \mathrm{fs}$ duration laser pulse. The interferometer was also used for temporal and spatial alignment of the two laser beams. Horizontal alignment was performed using plasma recombination radiation from the top view of the interaction point.

The measured electron charge with the colliding beam on or off is shown in Fig. 5. As can be seen, the colliding laser beam enhanced the amount of collected charge. Charge or $\gamma$-ray yield enhancement did not however show sensitive dependence (on the ps-scale) on the time delay between the two beams. This may be a result of the rather rough time scan, as well as the low signal-to-noise $(\mathrm{S} / \mathrm{N})$ ratio between electrons generated by wavebreaking and CPI. To elucidate the origin of the enhancement, a new version of the CPI experiment has been implemented that will use a preformed plasma channel generated by the ignitor-heater method $[15,12]$ in conjunction with a slit nozzle (200 $\mu \mathrm{m}$ wide by $4 \mathrm{~mm}$ long) gas jet. The original CPI scheme relied on operation in the standard laser wakefield regime [29]. In this regime, self-trapping is not expected but a preformed plasma channel is required since pulses will not self-guide [49]. As has been discussed, laser guiding in plasma channels can be operated in a regime that does not result in self-trapped electron beam acceleration, making a "clean" colliding pulse experiment possible. In addition, a magnetic spectrometer, with large momentum acceptance (spanning from $80 \mathrm{MeV}$ to $1 \mathrm{GeV}$ in a single shot at maximum excitation current), will be used to characterize the electron energy distribution of the bunches in a single-shot.

\section{BUNCH DURATION MEASUREMENT USING COHERENT TRANSITION RADIATION}

For nearly a decade, the L'OASIS group has devoted significant effort to the development of advanced beam diagnostics, that are capable of measuring the properties of electron beams in both transverse and longitudinal phase space. A detailed overview was written of the various diagnostic techniques that we have developed, and the physics behind them [50]. Some of the novel diagnostics discussed in Ref. [50] have the potential to resolve fs-bunches. Recently, the generation of coherent radiation from the electron bunches as they exit the plasma was studied experimentally $[8,51]$ and theoretically [9], both as a radiation source and as a diagnostic for measuring the electron bunch properties [36]. The interest in using these bunches for generation of coherent radiation is motivated by two components: (1) extremely dense, sub-ps electron bunches produced 


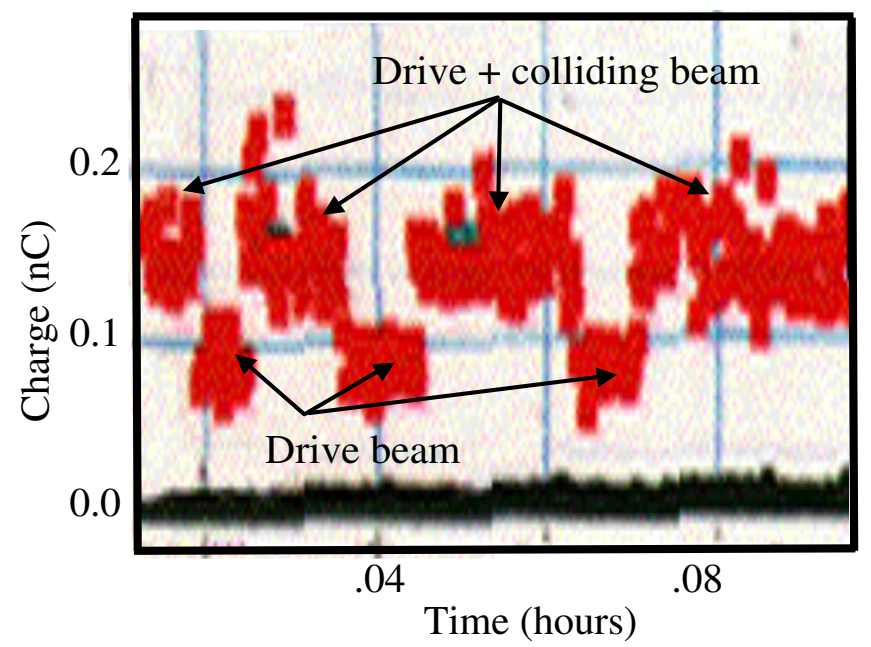

FIGURE 5. Enhanced electron yield by colliding laser beam. Measured charges are shown with and without the colliding beam.

with a compact laser-plasma accelerator and (2) the production of coherent transition radiation by these bunches at the boundary between a plasma and vacuum. Modeling of the performance of this source indicates that it has the potential for generating up to tens of $\mu \mathrm{J}$ per pulse [9], which is several orders of magnitude beyond that of conventional $\mathrm{THz}$ radiation sources.

The first experiments on the use of this radiation for bunch length measurements, were obtained by using a liquid helium cooled bolometer and a bandpass filter, to obtain coarse information about the radiated spectrum. Electron bunches were generated using a single intense laser beam focused on a He-backed gas jet [8]. The total radiated energy was collected in the $0.3-19 \mathrm{THz}$ and in the 0.3-2.9 THz ranges (and hence 2.9-19 THz range). Analysis of the raw experimental data (averaging of several tens of shots) shows that $22 \%$ of the observed radiation was emitted in the frequency range $0.3-2.9 \mathrm{THz}$, while $78 \%$ of the radiation was emitted within the frequency range $2.9-19 \mathrm{THz}$ range. To model this data, the radiated spectrum was calculated including diffraction $[51,9]$ from a finite radius plasma column. The spectrum exhibits a short wavelength cut-off due to the longitudinal coherence effects $\left(\lambda_{\min } \sim \sigma_{z}\right)$, and a long wavelength cut-off due to the influence of diffraction radiation from the transverse edge of the plasma $\left(\lambda_{\max } \sim \rho / u_{\mathrm{T}}\right)$.

Assuming a gaussian distribution with $\sigma_{z}=13.5 \mu \mathrm{m}$ and a Boltzmann energy distribution with a temperature $u_{\mathrm{T}}=9$, and a plasma with a transverse half-width of $100 \mu \mathrm{m}$, the calculated and measured spectrum contained the same amount of energy in the frequency range 0.3 to $2.9 \mathrm{THz}$, and 2.9 to $19 \mathrm{THz}$ range. An rms bunch length on the order of $10 \mu \mathrm{m}$, is consistent with self-modulated LWFA simulations [52], which typically predict electron beam bunch lengths to be on the order of the laser pulse duration or shorter.

A detailed spectrum (shown in Fig. 6) was obtained by using a scanning interferometer and, subsequently, Fourier transforming and smoothing the data using built-in 


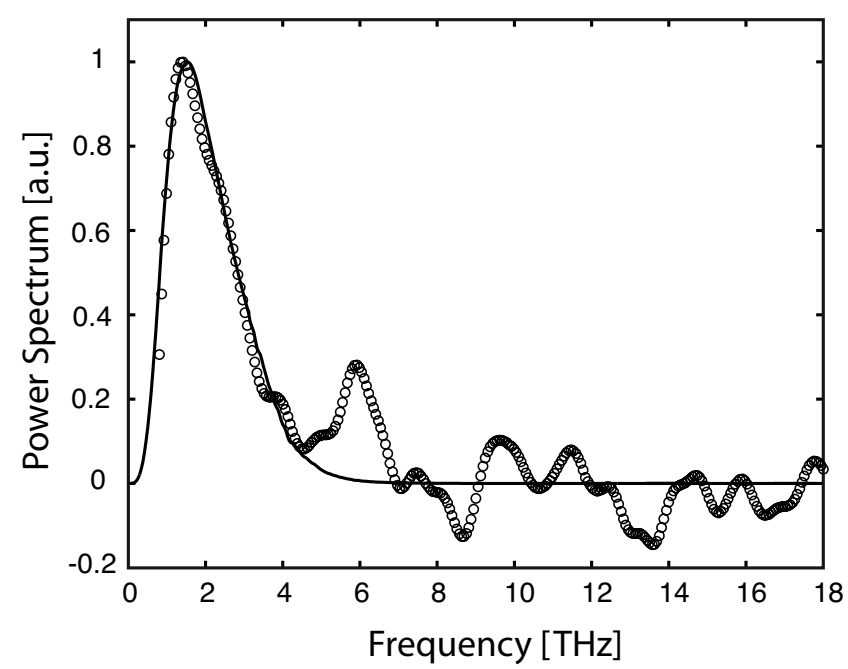

FIGURE 6. The Fourier transform of an interferogram (equivalent to the coherent power spectrum) of the coherently radiated $\mathrm{THz}$ emission by a laser accelerated electron bunch crossing the plasma-vacuum boundary. The interferogram was obtained using a scanning Michelson interferometer. The solid line is a best fit based on theory, after varying the parameters for the transverse plasma size $\rho$ and the electron bunch length $\sigma_{z}$.

software [36]. The black solid line shows a best fit to the spectrum applying the model described in Ref. $[9,51,36]$ and using $\rho=500 \mu \mathrm{m}$, and $\sigma_{z}=15 \mu \mathrm{m}$ (or $50 \mathrm{fs}$ ) for an observation angle $\theta=0.16 \mathrm{rad}( \pm 0.01 \mathrm{rad})$. The effects of the transmission curves of the various optical components, as well as the spectral response of the interferometer are included in this best fit. Whereas the bunch duration is consistent with the value obtained using the bandpass method, the transverse size is larger. This discrepancy is still under investigation.

A single shot diagnostic based on the electro-optic Pockel's effect is now being implemented to study the bunch duration for an individual pulse produced by the laser accelerator and is expected to enable detailed parametric studies of bunch duration versus plasma and laser properties, as well as bunch energy and propagation.

\section{SUMMARY AND FUTURE DEVELOPMENTS}

During the past few years, two important milestones towards the development of a laser driven accelerator have been achieved: laser guiding and production of mono-energetic beams. Using preformed plasma channels, laser beams of relativistic intensities (i.e., a $>1$ ) have been guided over 10 or more Rayleigh lengths [12]. Two regimes where found at LBNL. In the first regime, intense laser pulses at the 4 TW power level were guided and transmission levels approaching 50 percent were obtained. No significant numbers of accelerated electrons were detected. This result shows that it is possible to guide laser pulses over macroscopic distances at relativistic intensities without generating selftrapped electrons or 'dark current'. This is an important result for future development of laser accelerator modules. 
In the second regime, intense laser pulses were guided and high energy electron beams were observed that had an energy distribution with structure. Using a magnetic spectrometer, isolated intense mono-energetic beams were produced at the $100 \mathrm{MeV}$ energy level with energy spread at the level of a few percent, containing several 100's of picoCoulomb of charge. Transversely, these beam-lets were found to have a normalized emittance of 1-2 $\pi \mathrm{mm}$-mrad, limited by the resolution of the diagnostic. In this regime, the transmitted laser beam was severely depleted.

Narrow energy beams were also observed using $20 \mu \mathrm{m}$ scale spot size for the laser beams with long, lower density plasmas (LOA, RAL) or $6 \mu \mathrm{m}$ laser spots and short, higher density plasmas (LBNL unguided) or longer plasmas (LBNL guided). The fundamental reason for the observation of these narrow energy spread beams seems consistent to matching the acceleration distance to the dephasing distance of an electron in the plasma wave bucket. This was confirmed via particle-in-cell simulations using VORPAL [47] and via experiments at LBNL in which a single beam was used in gas jets of various lengths and gas densities.

\subsection{Where do we go from here?}

The immediate challenge ahead is to stabilize the performance of the accelerator. The charge per bunch is found to be stable, at the few percent level, in the high repetition LBNL experiments. The energy gain however fluctuates. This will require sophisticated control over the electron trapping process as well as the laser and plasma source operation. Since the energy depends linearly on laser intensity (see Eqn. 3), pulse length, pulse energy and spot size control is needed at the few percent level. Pulse length control requires spectral and pointing control. In the laser pulse stretcher and compressor, for example, pointing fluctuations translate into pulse duration fluctuation. To minimize pointing fluctuations, great care must be taken during the construction of future systems, to precisely control the environment (temperature and vibration). Pointing errors can also translate into pulse energy changes, due to changes in overlap between the gain medium and the amplified pulse. Novel methods for controlling laser pulse energy are being developed in industry, for short pulse systems, that meet our requirements. The origin of spot size fluctuations is not entirely understood, but may also be affected by pump laser changes that can affect the divergence, and overall spot size of the amplified beam (as well as beam pointing).

Plasma densities must be controlled at the percent level to ensure that the wake amplitude $\left(\propto n^{1 / 2}\right)$, dephasing length $\left(\propto 1 / n^{3 / 2}\right)$ and energy gain $(\propto 1 / n)$ remain constant. In addition to the development of plasma density diagnostics, capable of providing this level of accuracy, efforts are required to engineer pulsed gas jet systems that limit the shot-to-shot variation. When using statically filled chambers, such as in the case of a hydrogen based capillary discharge system [20], gas densities should be controllable to a sufficient level. This should result in reproducible plasma densities, provided the electrical discharge is reproducible.

As has been discussed, matching the accelerator length to the dephasing length can 
produce narrow energy spread beams, even when relying on self-trapping. However, accurate control of the performance of the accelerator, will most likely require a method for triggering the trapping process. Experiments are underway to study laser triggered injection, and are predicted by theory to produce narrow energy spread bunches.

Combined with channeling technology, this, we believe, then forms the basis of an all-optical accelerator that is expected to deliver GeV-class electron beams. As can be seen from the basic scaling laws, reducing the density and lengthening the distance over which the plasma channel extends is essential in enabling the development of compact $\mathrm{GeV}$ modules. To achieve multi-cm scale plasma channels, novel methods relying on hydrogen capillary discharges developed by Hooker et al. [20] will be tested with the 100 TW L'OASIS amplifier. To reduce the amount of energy required from the laser system in schemes that use inverse Bremsstrahlung to heat the plasmas (hydrodynamically formed channels), cluster jets will be tested such as being developed by Milchberg et al. [53].

The next challenge will be to go beyond $\mathrm{GeV}$ energy levels, requiring a demonstration of staging different modules, as well as development of laser systems capable of delivering high peak power, and large amounts of energy, at high repetition rate (i.e., high average power). As a simple example, consider producing a $10 \mathrm{GeV}$ electron beam, containing $1 \mathrm{nC}$ of charge $\left(6 \times 10^{9}\right.$ electrons $)$. This represents $10 \mathrm{~J}$ worth of energy and assuming a laser to particle beam efficiency between 1-10\%, requires therefore 100$1000 \mathrm{~J} /$ pulse of laser energy. It is obvious from this simple calculation, that it is essential for both accelerator and laser development to be pursued, if one wants to realize the goal of all-optical linacs. Such an accelerator holds the promise of offering unique beams, having femtosecond duration and containing 100's of $\mathrm{pC}$ of charge, with an emittance that equals or surpasses conventional linacs. If the development continues to be successful, it will serve as a compact multi-GeV injector for high energy physics applications, as well as the basis for novel radiation sources.

\section{ACKNOWLEDGMENTS}

The work that is presented here is the result of the dedication, inspiration, ideas and hard work of many people. In particular I would like to thank all L'OASIS group members, past and present. The present team consists of: Michael Dickinson, Eric Esarey, Catalin Filip (University of Nevada, Reno), Gwenael Fubiani, Cameron Geddes, Estelle Michel (University of Nevada, Reno), Pierre Michel, Bob Nagler, Kei Nakamura, Dieter Schneider (LLNL), Carl Schroeder, Brad Shadwick, Don Syversrud, Jeroen van Tilborg, Csaba Toth, Joe Wallig, Nathan Ybarrolazza, as well as our collaborators Simon Hooker and Tony Gonsalves from Oxford University. A special thanks goes to Cameron for all the excellent work on the channel guiding experiments and particle-incell simulations, Gwenael for the colliding pulse and space charge theory, Jeroen for the $\mathrm{THz}$ experimental work and Kei for his contributions to the colliding pulse experiment and the magnetic spectrometer. I also want to thank John Cary, David Bruhwiler, Dimitre Dimitrov and Chet Nieter for providing us VORPAL and working on the simulations. Thanks also goes to Gerry Dugan for his help in the magnet spectrometer design and 
space charge calculations, Ji Qiang and Rob Ryne for simulation support. I also wanted to thank Chuck Shank and Bill Barletta for their support and encouragement. Finally, I would like to thank the AAC2004 organizing committee for having given me the opportunity to summarize (and giving my own personal view of) our exciting field of laser acceleration. This work was supported by the Department of Energy, Office of Science, High Energy Physics under contract DE-AC03-76SF0098.

\section{REFERENCES}

1. Modena, A., Najmudin, Z., Dangor, A. E., Clayton, C. E., Marsh, K. A., Joshi, C., Malka, V., Darrow, C. B., Danson, C., Neely, D., and Walsh, F. N., Nature, 377, 606-608 (1995).

2. Umstadter, D., Chen, S.-Y., Maksimchuk, A., Mourou, G., and Wagner, R., Science, 273, 472-5 (1996).

3. Leemans, W. P., Catravas, P., Esarey, E., Geddes, C. G. R., Toth, C., Trines, R., Schroeder, C. B., Shadwick, B. A., van Tilborg, J., and Faure, J., Phys. Rev. Lett., 89, 4802 (2002).

4. Schroeder, C. B., Esarey, E., Geddes, C. G. R., Tóth, C., Shadwick, B. A., van Tilborg, J., Faure, J., and Leemans, W. P., Phys. Plasmas, 10, 2039-2046 (2003).

5. Santala, M. I. K., Najmudin, Z., Clark, E. L., Tatarakis, M., Krushelnick, K., Dangor, A. E., Malka, V., Faure, J., Allott, R., and Clarke, R. J., Phys. Rev. Lett., 86, 1227-1230 (2001).

6. Leemans, W. P., Rodgers, D., Catravas, P., Geddes, C. G. R., Fubiani, G., Esarey, E., Shadwick, B., Donahue, R., and Smith, A., Phys. Plasmas, 8, 2510-2516 (2001).

7. Ledingham, K. W. D., McKenna, P., and Singhal, R. P., Science, 300, 1107 (2003).

8. Leemans, W. P., Geddes, C. G. R., Faure, J., Tóth, C., van Tilborg, J., Schroeder, C. B., Esarey, E., Fubiani, G., Auerbach, D., Marcelis, B., Carnahan, M. A., Kaindl, R. A., Byrd, J., and Martin, M., Phys. Rev. Lett., 91, 074802 (2003).

9. Schroeder, C. B., Esarey, E., van Tilborg, J., and Leemans, W. P., Phys. Rev. E, 69, 016501 (2004).

10. Malka, V., Fritzler, S., Lefebvre, E., Aleonard, M. M., Burgy, F., Chambaret, J. P., Chemin, J. F., Krushelnick, K., Malka, G., Mangles, S. P. D., Najmudin, Z., Pittman, M., Rousseau, J. P., Scheurer, J. N., Walton, B., and Dangor, A. E., Science, 298, 1596-1600 (2002).

11. Krushelnick, K., CLEO 2004, paper JTuA2.

12. Geddes, C. G. R., Tóth, C., van Tilborg, J., Esarey, E., Schroeder, C. B., Bruhwiler, D., Nieter, C., Cary, J., and Leemans, W. P., Nature (2004), to be published.

13. Leemans, W. P., Siders, C. W., Esarey, E., Andreev, N. E., Shvets, G., and Mori, W. B., IEEE Trans. Plasma Sci., 24, 331-342 (1996).

14. Esarey, E., Sprangle, P., Krall, J., and Ting, A., IEEE Trans. Plasma Sci., 24, 252-288 (1996).

15. Volfbeyn, P., Esarey, E., and Leemans, W. P., Phys. Plasmas, 6, 2269-2277 (1999).

16. Alexeev, I., Antonsen, Jr., T. M., Kim, K. Y., and Milchberg, H. M., Phys. Rev. Lett., 90, 103402 (2003).

17. Downer, M. C., Chiu, C., Fomyts'kyi, M., Gaul, E. W., Grigsby, F., Matlis, N. H., Shim, B., Smith, P. J., and Zgadzaj, R., "Plasma Channels and Laser Pulse Tailoring for GeV Laser-Plasma Accelerators," in Advanced Accelerator Concepts. Eleventh Workshop, 2003, vol. 647, p. 654.

18. Tochitsky, S. Y., Narang, R., Filip, C. V., Musumeci, P., Clayton, C. E., Yoder, R. B., Marsh, K. A., Rosenzweig, J. B., Pellegrini, C., and Joshi, C., Phys. Rev. Lett., 92, 095004 (2004).

19. Sprangle, P., Hafizi, B., Peñano, J. R., Hubbard, R. F., Ting, A., Moore, C. I., Gordon, D. F., Zigler, A., Kaganovich, D., and Antonsen, Jr., T. M., Phys. Rev. E., 63, 056405 (2001).

20. Spence, D. J., and Hooker, S. M., Phys. Rev. E, 63, 015401(R) (2001).

21. Zhidkov, A., Koga, J., Esirkepov, T., Hosokai, T., Uesaka, M., and Tajima, T., Phys. Rev. E, 69, 066408 (2004).

22. Lopes, N. C., Figueira, G., Silva, L. O., Dias, J. M., Fonseca, R., Cardoso, L., Russo, C., Carias, C., Mendes, G., Vieira, J., and Mendonça, J. T., Phys. Rev. E, 68, 035402 (2003).

23. Xiao, Y.-F., Chu, H.-H., Tsai, H.-E., Lee, C.-H., Lin, J.-Y., Wang, J., and Chen, S.-Y., Phys. Plasmas, 11, L21-L24 (2004). 
24. Cros, B., Courtois, C., Matthieussent, G., Bernardo, A. D., Batani, D., Andreev, N., and Kuznetsov, S., Phys. Rev. E, 65, 026405 (2002).

25. Geddes, C. G. R., Toth, C., Tilborg, J. V., Esarey, E., Schroeder, C. B., Bruhwiler, D., Cary, J., and Leemans, W. P., "Laser guiding at relativistic intensities and wakefield particle acceleration in plasma channels", in these Proceedings.

26. Geddes, C. G. R., Tóth, C., van Tilborg, J., Esarey, E., Schroeder, C. B., Bruhwiler, D., Nieter, C., Cary, J., and Leemans, W. P., Phys.Rev.Lett. (2004), submitted.

27. Moore, C. I., Ting, A., McNaught, S. J., Qiu, J., Burris, H. R., and Sprangle, P., Phys. Rev. Lett., 82, 1688-1691 (1999).

28. Umstadter, D., Kim, J. K., and Dodd, E., Phys. Rev. Lett., 76, 2073-6 (1996).

29. Esarey, E., Hubbard, R. F., Leemans, W. P., Ting, A., and Sprangle, P., Phys. Rev. Lett., 79, 2682-2685 (1997).

30. Schroeder, C. B., Lee, P. B., Wurtele, J. S., Esarey, E., and Leemans, W. P., Phys. Rev. E, 59, 60376047 (1999).

31. Fubiani, G., Esarey, E., Schroeder, C. B., and Leemans, W. P., Physical Review E, 70, 016402 (2004).

32. Nakamura, K., Fubiani, G., Geddes, C. G. R., Michel, P., van Tilborg, J., Tóth, C., Esarey, E., Schroeder, C. B., and Leemans, W. P., "Laser Triggered Injection of Electrons in a Laser Wakefield Accelerator with the Colliding Pulse Method," in Advanced Accelerator Concepts. Eleventh Workshop, edited by V. Yakimenko, AIP Conf. Proc., Amer. Inst. Phys., New York, 2005.

33. Siders, C. W., Leblanc, S. P., Fisher, D., Tajima, T., Downer, M. C., Babine, A., Stepanov, A., and Sergeev, A., Phys. Rev. Lett., 76, 3570-3 (1996).

34. Siders, C. W., Leblanc, S. P., Babine, A., Stepanov, A., Sergeev, A., Tajima, T., and Downer, M. C., IEEE Trans. Plasma Sci., 24, 301-315 (1996).

35. Shah, R., Banerjee, S., Valenzuela, A., Korbiak, K., Maksimchuk, A., and Umstadter, D., Bull. Am. Phys. Soc., 48, 252 (2003).

36. van Tilborg, J., Geddes, C. G. R., Toth, C., Esarey, E., Schroeder, C. B., Martin, M. C., Hao, Z., and Leemans, W. P., "Coherent transition radiation from a laser wakefield accelerator as an electron bunch diagnostic", in these Proceedings.

37. Fritzler, S., Lefebvre, E., Malka, V., Burgy, F., Dangor, A. E., Krushelnick, K., Mangles, S. P. D., Najmudin, Z., Rousseau, J.-P., and B.Walton, Phys. Rev. Lett., 92, 165006 (2004).

38. Fubiani, G., Dugan, G., Leemans, W., Esarey, E., and Bobin, J. L., "Semi-analytical 6D space charge model for dense electron bunches with large energy spreads," in Proc. of the 2002 Advanced Accelerator Concepts Workshop, edited by C. E. Clayton and P. Muggli, AIP, NY, 2002, vol. 647, pp. 203-212.

39. Fubiani, G., Qiang, J., Leemans, W. P., Schroeder, C. B., Esarey, E., and Dugan, G. (2004), in preparation.

40. Yakimenko, V., Babzien, M., Ben-Zvi, I., Malone, R., and X.-J.Wang, Phys. Rev. ST Accel. Beams, 6, 122801 (2003).

41. Tóth, C., Geddes, C. G. R., van Tilborg, J., and Leemans, W. P., "A multi-beam, multi-terawatt ti:sapphire laser system for laser wake-field acceleration studies", in these Proceedings.

42. Durfee III, C. G., and Milchberg, H. M., Phys. Rev. Lett., 71, 2409-2411 (1993).

43. Milchberg, H. M., Clark, T. R., Durfee III, C. G., and Antonsen, Jr., T. M., Phys. Plasmas, 3, 21492155 (1996).

44. Esarey, E., Schroeder, C. B., Shadwick, B. A., and Leemans, W. P., "Non-linear pump depletion and electron dephasing in laser wakefield accelerators", in these Proceedings.

45. Rankin, R., Capjack, C. E., Burnett, N. H., and Corkum, P. B., Optics Letters, 16, 835-837 (1991).

46. Leemans, W. P., Clayton, C. E., Mori, W. B., Marsh, K. A., Kaw, P. K., Dyson, A., Joshi, C., and Wallace, J. M., Phys. Rev. A, 46, 1091-1105 (1992).

47. Nieter, C., and Cary, J., J. Comp. Phys., 196, 448 (2004).

48. Pukhov, A., and Meyer-ter-Vehn, J., Appl. Phys. B, 74, 355 (2002).

49. Sprangle, P., Esarey, E., Krall, J., and Joyce, G., Phys. Rev. Lett., 69, 2200-2203 (1992).

50. Catravas, P., Esarey, E., and Leemans, W. P., Phys. Plasmas, 9, 2428-2436 (2002).

51. Leemans, W. P., van Tilborg, J., Faure, J., Geddes, C. G. R., Tóth, C., Schroeder, C. B., Esarey, E., and Fubiani, G., Phys. Plasmas, 11, 2899-2906 (2004).

52. Tzeng, K. C., Mori, W. B., and Katsouleas, T., Phys. Rev. Lett., 79, 5258-5261 (1997).

53. Taguchi, T., Antonsen, Jr., T. M., and Milchberg, H. M., Phys. Rev. Lett., 92, 205003 (2004). 